\title{
Recent applications of the subtracted SRPA approximation
}

\author{
Danilo Gambacurta ${ }^{1, *}$ \\ ${ }^{1}$ Extreme Light Infrastructure - Nuclear Physics (ELI-NP), Horia Hulubei National Institute for Physics and Nuclear Engineering, 30 \\ Reactorului Street, RO-077125 Magurele, Jud. Ilfov, Romania
}

\begin{abstract}
The Second Random Phase Approximation (SRPA) is a natural extension of the Random Phase Approximation obtained by introducing more general excitation operators where two particle-two hole configurations, in addition to the one particle-one hole ones, are considered. Only in the last years, large-scale SRPA calculations, without usually employed approximations have been performed. The SRPA model corrected by a subtraction procedure designed to cure double counting issues and the related instabilities has been recently implemented and applied in the study of different physical cases. We report here on some of the most recent results obtained by using this model. In particular, results on the dipole strength ${ }^{48} \mathrm{Ca}$ and on a systematic study of the isoscalar giant quadrupole resonance in spherical nuclei will be shown and discussed.
\end{abstract}

\section{Introduction}

The random-phase-approximation (RPA) model provides a microscopic description of the nuclear collective excitations constructed as superpositions of 1 particle- 1 hole (1p1h) configurations. This approach is able to provide the global features of Giant Resonances (GR) such as the centroid energy, the total strength and the corresponding energy-weighted sum rules. However, it is not suited to describe other important properties as for example the GR's fine structure and the spreading width, generated by the coupling between $1 \mathrm{p} 1 \mathrm{~h}$ configurations with more complex degrees of freedom. Approaches aimed at the description of this coupling are for example the particle-phonon coupling models based on the so-called time-blocking approximation $[1,2]$ and the relativistic quasiparticle timeblocking approxi- mation [3, 4], the quasiparticle-phonon model $[5,6]$, particle-phonon (or quasiparticle-phonon) coupling models $[7,8]$.

The Second RPA (SRPA) model is a natural extension of RPA where a more general description of the nuclear excitations is considered and provides a valuable tool for the prediction of spreading widths and fine structure properties due to the introduction of 2 particle- 2 hole $(2 \mathrm{p} 2 \mathrm{~h}$ ) configurations [9-12]. The SRPA model has been recently improved by using the so called subtraction procedure $[13,14]$ designed to handle the problem of the double counting of correlations within energy-density-functional (EDF) based model which go beyond the RPA level. This procedure cures some of the drawbacks and the limitations of the SRPA model formulated in the EDF framework providing thus a robust and stable theoretical tool for a beyond-mean-field description of the excitation spectra of many-body systems. In this work, we briefly report about some recent applications of the SRPA model based

*e-mail: danilo.gambacurta@eli-np.ro on the subtraction procedure that we will refer at as SSRPA.

In Section 2, we analyze the dipole response in ${ }^{48} \mathrm{Ca}$. In particular we show how the SSRPA provides a satisfactory description of the low-lying dipole spectrum (below $10 \mathrm{MeV}$ ) and of the giant dipole resonance (GDR).

In Section 3 we present the systematic trends obtained for the centroids and the widths in the SSRPA model for the isoscalar giant quadrupole resonances for different nuclei, from ${ }^{30} \mathrm{Si}$ to ${ }^{208} \mathrm{~Pb}$. In the same section we also show a detailed comparison of the strength distributions for ${ }^{90} \mathrm{Zr}$. Comparison with the RPA results and with experimental spectra are also shown. Finally in Section 4 some conclusions are drawn.

\section{Dipole response in ${ }^{48} \mathrm{Ca}$}

Details on the SRPA and SSRPA formalism can be found in Refs $[10,11,13]$. In the following analysis, we used a cutoff of $100 \mathrm{MeV}$ in building the 1ph configurations, ensuring a full preservation of the isoscalar and isovector Energy Weighted Sum Rules (EWSRs). Results are illustrated with two Skyrme parametrizations, SGII [15] and SLy4 [16] and more detailes can be found in Ref. [17]. Deviations of less than $1 \%$ are found in both cases in RPA. In the SRPA and SSRPA calculations, a cutoff of $60 \mathrm{MeV}$ in the $2 \mathrm{p} 2 \mathrm{~h}$ configurations is used. The dipole response below the neutron threshold in ${ }^{48} \mathrm{Ca}$ has been measured with the $\left(\gamma, \gamma^{\prime}\right)$ reaction [18]. The RPA predictions fail in reproducing such a low-lying strength either because the lowest RPA energies are higher than $10 \mathrm{MeV}$ or because the RPA model cannot provide the observed fragmentation. A fragmented SRPA dipole strength below $10 \mathrm{MeV}$ was indeed found [11]. However, the SRPA $B(E 1)$ transition probability, integrated up to $10 \mathrm{MeV}$, was found to be definitely much larger than the experimental value. We 
show here that problem of overestimation of the transition probability is strongly reduced in SSRPA, providing thus a rather good description of the experimental data. Some states having strong $1 \mathrm{ph}$ components, which are too much shifted downwards by the standard SRPA, are pushed to higher energies by the subtraction, reducing in this way the $B(E 1)$ value below $10 \mathrm{MeV}$.

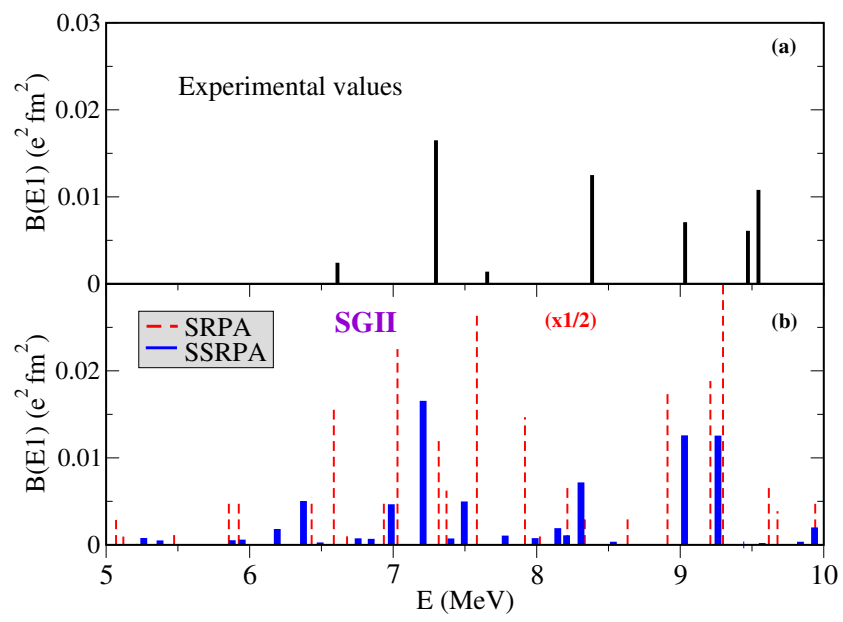

Figure 1. Low-lying dipole strength in ${ }^{48} \mathrm{Ca}$ : (a) Experimental $B(E 1)$ values [18]; (b) Theoretical predictions for the transition probabilities $B(E 1)$ calculated with the standard SRPA (dashed red bars; the values have been divided by 2) and with the SSRPA (blue thick bars).

In Figure 1(a) we show the experimental transition probabilities $B(E 1)$ [18]. The corresponding theoretical results are displayed in Figure 1(b). One observes that the total strength provided by the standard SRPA is much higher than the experimental one as already found and discussed [12]. The theoretical prediction given by the SSRPA model, on the other side, is in a very satisfactory agreement with respect to the experimental data. The fragmentation of the states follows quite well the experimental distribution in the energy position of the main peaks. The total $B E(1)$ integrated up to $10 \mathrm{MeV}$ is $0.126(0.078) \mathrm{e}^{2}$ $\mathrm{fm}^{2}$ in the SLy4 (SGII) case to be compared with the experimental value $0.068 \mathrm{e}^{2} \mathrm{fm}^{2}$.

We move now to the energy region between 10 and $25 \mathrm{MeV}$, where the GDR is located. Figure 2 shows the strength distributions calculated with the parametrization SGII in RPA, SRPA, and SSRPA, compared with the experimental distributions [19]. Results with the SGII interaction are shown but a similar behaviour is found also with SLy4. To make simpler the comparison, a folding with a Lorentzian having a width of $0.25 \mathrm{MeV}$ is performed. This folding produces some artificial spreading in the RPA case. For both SRPA and SSRPA, a more pronounced spreading is found corresponding to a physical width produced by the extremely dense distribution generated by the $2 \mathrm{p} 2 \mathrm{~h}$ configurations. It can be observed that the width is indeed in a satisfactory agreement with the experimental distribution. Experimental centroid energy $E_{C}$ and width $\Gamma$ are equal to $18.9 \pm 0.2$ and $3.9 \pm 0.4 \mathrm{MeV}$, respectively [19] We have computed the theoretical centroid energies and widths using the expressions

$$
E_{C}=\frac{m_{1}}{m_{0}}, \quad \Gamma_{C}=\sqrt{m_{2} / m_{0}-\left(m_{1} / m_{0}\right)^{2}}
$$

where $m_{k}$ represents the moment of order $k$ integrated in the energy region of interest.

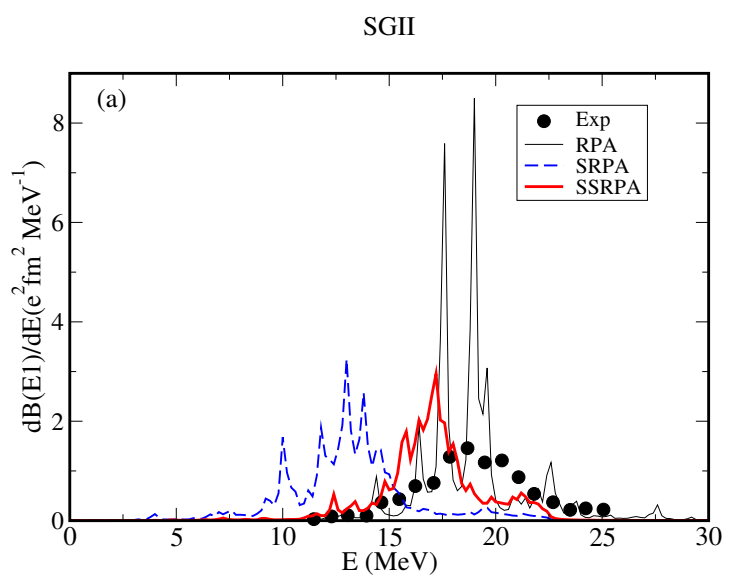

Figure 2. Dipole strength distributions in ${ }^{48} \mathrm{Ca}$ evaluated with RPA (solid black line), SRPA (blue dashed line), and SSRPA (thick red line), compared with the experimental distributions (black circles) from Ref. [19]. The SGII Skyrme interaction is used.

The theoretical centroid energies are 18.0, 13.4, and 16.2 MeV with RPA, SRPA, and SSRPA, respectively. The widths are equal to 2.5 and $2.1 \mathrm{MeV}$ with SRPA and SSRPA, respectively. The parametrization SLy4 provides less satisfactory results than the Skyrme interaction SGII. In particular, the SSRPA centroid energy is lower than the experimental centroid by more than $2.5 \mathrm{MeV}$. Figure 3 displays in the two panels the SSRPA strength distributions obtained with SGII (a) and SLy4 (b), shifted upwards respectively by 1.5 (a) and 2.7 (b) $\mathrm{MeV}$, together with the RPA strength distributions and the experimental data. This plot clearly illustrates how well the width of the resonance is described and shows the significant improvement with respect to RPA.

\section{Isoscalar GQR: a systematic study}

In this section we analyze the isoscalar GQR in several closed shell nuclei. More details can be found in Ref. [20]. It is quite clear, that the SRPA approach, especially if performed within the EDF approach, i.e. with the use of effective interactions, is plagued by double-counting and instabilities issues[21, 22]. We underline that these issues affects mainly the centroid of the strength distribution but not its width, so the SRPA and SSRPA widths are indeed very similar. However, as the SRPA centrids are quite far from th experimental ones, in the following we will show only the comparison between the RPA and SSRPA results. The SLy4 interaction is used. The single-particle space 


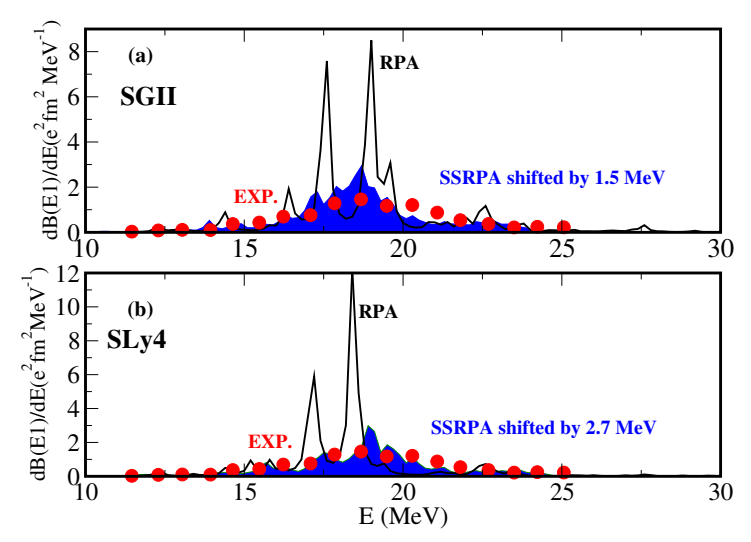

Figure 3. (a) SSRPA results for ${ }^{48} \mathrm{Ca}$ shifted by $1.5 \mathrm{MeV}$ (blue area) compared with the RPA strength (black line) and with the experimental values (red circles), obtained with the parametrization SGII. (b) Same as in (a) but the results are obtained with the parametrization SLy4 and the shift of the SSRPA spectrum is larger $(2.7 \mathrm{MeV})$.

is chosen large enough to assure that the EWSR are preserved within $1 \%$. For the $2 \mathrm{p} 2 \mathrm{~h}$ space in the SSRPA calculations, we use a cutoff of $60 \mathrm{MeV}$ for medium-mass nuclei (up to ${ }^{68} \mathrm{Ni}$ ) and of $50 \mathrm{MeV}$ for the heavy ones ( up to ${ }^{208} \mathrm{~Pb}$ ). We checked that these cutoff values provide stable results. Centroids and widths of the IS GQRs were computed by fitting a Lorentzian distribution. This adjustment was done on curves obtained by folding the discrete spectra with narrow Lorentzian distributions. In the SSRPA case, the folding done with very narrow distributions follows fairly well the extremely dense SSRPA spectra and no artificial effects induced by the performed folding are observed when the Lorentzian distribution is fitted. On the other side, in those RPA cases where there is a unique dominant peak, the width extracted in this way turns out to be equal to the width of the folding Lorentzian. We used in these cases very narrow folding Lorentzian (100-keV width) not to induce any artificial spreading effect.

The systematic trend provided by the SSRPA model for the centroids is shown in Fig. 4 where SSRPA results (blue diamonds) are compared with the corresponding RPA results (magenta triangles) and with the experimental data (black circles) represented in the figure with the corresponding error bars. Nuclei for which a comparison between our theoretical predictions and the corresponding experimental data may be done are identified in the figure by vertical dotted red lines. We observe that the SSRPA centroids are systematically located at lower energies than the RPA values. For most of the cases where the experimental centroids are available, we observe that the SSRPA energies are in better agreement with the experimental values than the RPA centroids, which in general overestimate the data, as can be seen in Fig. 4.

We expect that the description of the widths is strongly modified in the SSRPA model, compared to the RPA case,

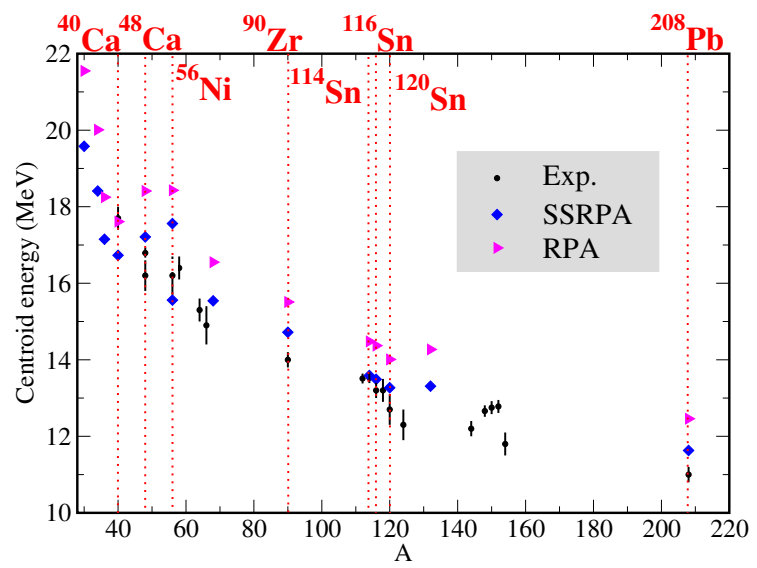

Figure 4. Centroids of the isoscalar GQR: the experimental data in black circles are compared with the SSRPA (RPA) predictions are plotted as blue diamonds (magenta triangles).

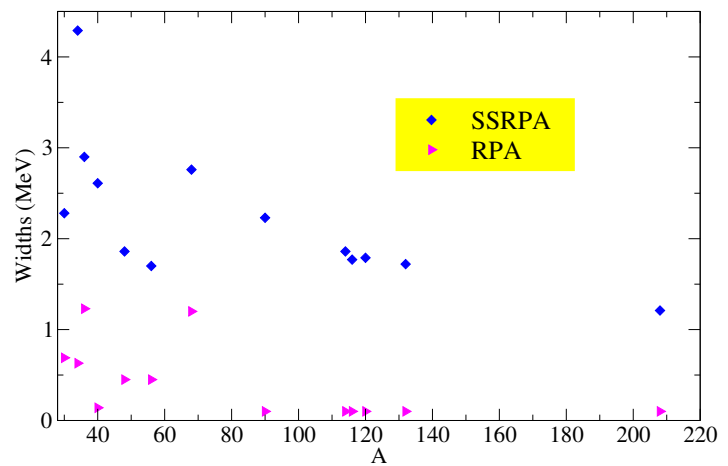

Figure 5. Theoretical widths calculated with the fit of a Lorentzian distribution within RPA and SSRPA models.

because an additional spreading effect is explicitly taken into account (in addition to the single-particle Landau damping which is already present in RPA) owing to the coupling between $1 \mathrm{p} 1 \mathrm{~h}$ and $2 \mathrm{p} 2 \mathrm{~h}$ configurations. Figure 5 displays the SSRPA and RPA widths. We specify once more that our predictions of the total widths contain the Landau-damping effect (RPA and SSRPA) and the spreading contribution (SSRPA). The escape width is missing. Even if the escape width is expected to be less important than the spreading width, it may modify some of our predictions, especially for light nuclei. We observe that the SSRPA widths are, as expected, systematically larger than the RPA ones.

As far as width, fine structure and fragmentation are concerned, we dedicate a more focused discussion to a specific case, namely ${ }^{90} \mathrm{Zr}$. High-resolution $\left(p, p^{\prime}\right)$ spectra are available [23]. Energy resolutions of $\sim 40 \mathrm{keV}$ could be achieved and the fine structure of the excitation spectra could be examined. We present in Fig. 6(c) the 


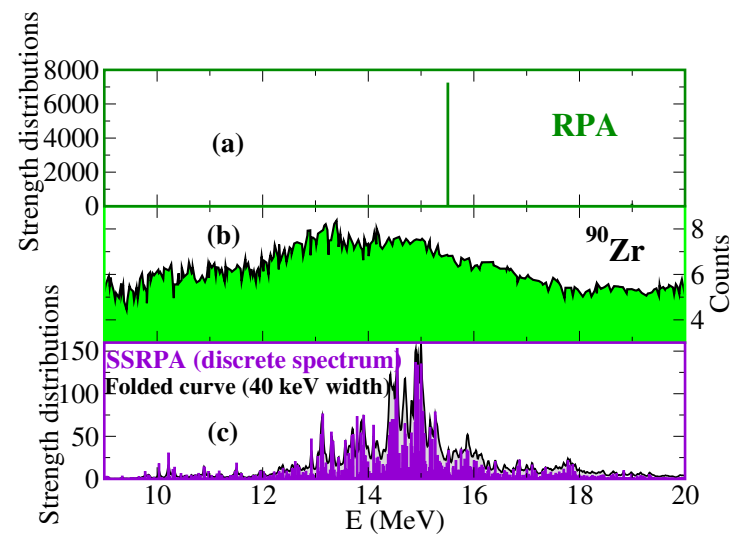

Figure 6. (a) RPA strength distributions, (b) Experimental spectrum [23] and (c) SSRPA strength distributions for the isoscalar GQR for ${ }^{90} \mathrm{Zr}$. For the cases of the RPA and SSRPA discrete spectra, units are $e^{2} \mathrm{fm}^{4}$. For the SSRPA folded case, units are $e^{2}$ $\mathrm{fm}^{4} \mathrm{MeV}^{-1}$.

SSRPA strength distribution (violet bars) for the nucleus ${ }^{90} \mathrm{Zr}$. To better compare it with the corresponding experimental spectrum (b), a folded curve is also plotted (black solid line and grey area), obtained by folding the discrete distribution with a Lorentzian of width equal to $40 \mathrm{keV}$, which corresponds to the experimental energy resolution. The RPA centroid is larger by more than $1 \mathrm{MeV}$ compared to the experimental value (Fig. 4). The SSRPA prediction is located at lower energies, in better agreement with data. We observe that the folded curve follows well the fine structure provided by the discrete spectrum. The RPA strength distribution is also shown in Fig. 6(a). Our RPA prediction displays a unique dominant peak, whereas the SSRPA strength distribution is much more fragmented and extends over a larger energy region where the experimental data are spread. We note however that the experimental strength is distributed in a broader region than the theoretical one. This is probably related to missing effects in our theoretical model, such as the inclusion of higher-order configurations or, to a less extent, to the coupling with the continuum which is neglected here.

\section{Conclusions}

We have discussed two recent applications of the SSRPA model. First we analyze the dipole response in ${ }^{48} \mathrm{Ca}$ and we show how the SSRPA is able to provide a better description with respect the RPA regarding both the lowlying excited states and the width of the GDR. Afterwards, we have shown a systematic analysis of the isoscalar GQR in several spherical nuclei. Also in this case the SSRPA ia able to provide a better description of the centroid energies and widths with respect the RPA. We also discussed the fine structure properties described by the SSRPA for the specific case of ${ }^{90} \mathrm{Zr}$. A significant improvement of the description of the spreading width is found in SSRPA compared to RPA (where a single dominant peak is predicted) and fragmented strengths are obtained.

\section{References}

[1] S.P. Kamerdzhiev, G.Y. Tertychny, V.I. Tselyaev, Phys. Part. Nucl. 28, 134 (1997).

[2] V.I. Tselyaev, Phys. Rev. C 75, 024306 (2007).

[3] E. Litvinova, P. Ring, and V. Tselyaev, Phys. Rev. C 78, 014312 (2008).

[4] I.A. Egorova and E. Litvinova, Phys. Rev. C 94, 034322 (2016).

[5] V.G. Soloviev, Theory of Atomic Nuclei: Quasiparticles and Phonons (Institute of Physics, Bristol and Philadelphia, 1992).

[6] A.P. Severyukhin, S. Aberg, N.N. Arsenyev, and R.G. Nazmitdinov, Phys. Rev. C 95, 061305(R) (2017).

[7] G. Colò, H. Sagawa, N. Van Giai, P.F. Bortignon, and T. Suzuki, Phys. Rev. C 57, 3049 (1998).

[8] Y.F. Niu, G. Colò, E. Vigezzi, C.L. Bai, and H. Sagawa, Phys. Rev. C 94, 064328 (2016).

[9] P. Papakonstantinou and R. Roth, Phys. Rev. C 81, 024317 (2010)

[10] D. Gambacurta, M. Grasso, and F. Catara, Phys. Rev. C 81, 054312 (2010)

[11] D. Gambacurta, M. Grasso, and F. Catara, Phys. Rev. C 84, 034301 (2011)

[12] D. Gambacurta, M. Grasso, V. De Donno, G. Co', and F. Catara, Phys. Rev. C 86, 021304(R), (2012)

[13] D. Gambacurta, M. Grasso, and J. Engel, Phys. Rev. C 92, 034303 (2015)

[14] D. Gambacurta and M. Grasso, Eur. Phys. J. A 52, 198 (2016)

[15] N. V. Giai, H.. Sagawa, Phys. Lett. B 106, 379 (1981); Nucl. Phys. A 371, 1 (1981)

[16] E. Chabanat, P. Bonche, P. Haensel, J. Meyer, R. Schaeffer, Nucl. Phys. A 627, 710 (1997); ibid A 635, 231 (1998); ibid 643, 441 (1998)

[17] D. Gambacurta, M. Grasso, and O. Vasseur, Phys. Lett. B 777, 163 (2018)

[18] T. Hartmann, J. Enders, P. Mohr, K. Vogt, S. Volz, and A. Zilges, Phys. Rev. C 65, 034301 (2002)

[19] J. Birkhan et al., Phys. Rev. Lett. 118, 252501 (2017)

[20] O. Vasseur, D. Gambacurta, and M. Grasso,Phys. Rev. C 98, 044313, (2018)

[21] V. I. Tselyaev, Phys. Rev. C 88, 054301 (2013).

[22] P. Papakonstantinou, Phys. Rev. C 90, 024305 (2014).

[23] A. Shevchenko, et al., Phys. Rev. Lett. 93, 122501 (2004) 